\title{
Factores socioeconómicos en la estimación de la huella hídrica personal: el caso de la Pontificia Universidad Católica del Ecuador
}

Socio-economic Factors in Estimating the Personal Water Footprint: the Case of the Pontificia Universidad Católica del Ecuador

Fatores socioeconômicos na estimativa da pegada hídrica individual: o caso da Pontificia Universidad Católica del Ecuador

\author{
Pablo Chafla Martínez \\ Pontificia Universidad Católica del Ecuador. Quito, Ecuador \\ pchafla328@puce.edu.ec \\ https://orcid.org/0000-0002-7182-7131 \\ Juan Chafla Altamirano \\ Pontificia Universidad Católica del Ecuador. Quito, Ecuador \\ jchafla390@puce.edu.ec \\ https://orcid.org/0000-0002-0773-6745 \\ Andrea Mancheno Corral \\ Pontificia Universidad Católica del Ecuador. Quito, Ecuador \\ ramancheno608@puce.edu.ec. \\ https://orcid.org/0000-0002-8698-3786
}

DOI: https://doi.org/10.32719/25506641.2021.9.8

Recibido: 22 de mayo de 2020 • Revisado: 12 de julio de 2020 • Aceptado: 11 de septiembre de 2020 Artículo de investigación

Licencia Creative Commons

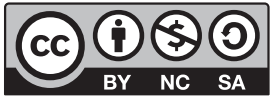




\section{Resumen}

El presente estudio tiene como objetivo determinar la existencia de correlaciones entre la huella hídrica personal (HH) estimada de manera volumétrica (metros cúbicos/persona), con determinadas variables socioeconómicas como el sexo, la edad, el ingreso familiar, el nivel de educación, el tipo y los años de la vivienda y el número de personas con las que comparten la vivienda. Con una metodología de investigación descriptiva y exploratoria, se realizó una encuesta virtual a finales de 2019 a toda la comunidad universitaria de la Pontificia Universidad Católica del Ecuador (docentes, administrativos y alumnos) en la sede principal de la ciudad de Quito. También se dejó abierta la posibilidad de que personas fuera de la universidad pudieran llenar la encuesta y de esta manera poder evaluar si el consumo de los miembros de la comunidad universitaria que habían recibido información sobre uso sustentable del agua, presentaba alguna diferencia con las personas que no la habían recibido. Se encontró que existe una correlación significativa entre la $\mathrm{HH}$ y algunas de las variables socioeconómicas analizadas como el ingreso familiar, el sexo y el nivel educativo. Se desprende también de la investigación que la $\mathrm{HH}$ puede ser utilizada como un buen indicador agregado de consumo de agua que ayude a tomar conciencia sobre patrones insostenibles de consumo, mas no es recomendable su utilización como instrumento para la gestión y planificación hidrológica por la concentración y sencillez de la información que presenta.

Palabras clave: estimación económica, huella hídrica personal, correlaciones de variables socioeconómicas, indicador agregado.

JEL: Q5 Economía del medioambiente.

\section{Abstract}

The present study aims to determine the existence of correlations between the personal water footprint $(\mathrm{HH})$ estimated in a volumetric way (cubic meters/person), with certain socio-economic variables such as sex, age, family income, level of education, the type and age of the dwelling and the number of people with whom they share the dwelling. With a descriptive and exploratory research methodology, a virtual survey was conducted at the end of 2019 to the entire university community of the Pontificia Universidad Católica del Ecuador (teachers, administrators and students) at the main headquarters of Quito. It was also left open the possibility that people from outside the university could fill out the survey and in this way be able to assess whether the consumption of people from the university community who had received information on sustainable water use presented any difference with those who they had not received it. It was found that there is a significant correlation between $\mathrm{HH}$ and some of the socioeconomic variables analysed, such as family income, sex and educational level. It is also clear from the research that $\mathrm{HH}$ can be used as a good aggregate indicator of water consumption that helps to become aware of unsustainable patterns of consumption, and not being used as an instrument for hydrological management and planning due to concentration and simplicity of information it presents.

Keywords: Economic estimation, personal water footprint, correlations of socioeconomic variables, aggregate indicator.

JEL: Q5 Economics of the environment. 


\section{Resumo}

O presente estudo tem por objetivo determinar a existência de correlações entre a pegada hídrica individual $(\mathrm{PH})$ estimada de maneira volumétrica (metros cúbicos/pessoa) e determinadas variáveis socioeconômicas como sexo, idade, renda familiar, nível educacional, tipo e anos de moradia e número de pessoas com as quais a mesma é compartilhada. A partir de uma metodologia de pesquisa descritiva e exploratória, realizou-se uma enquete virtual no final de 2019 dirigida a toda a comunidade universitária da Pontifícia Universidade Católica do Equador (docentes, corpo administrativo e alunos) na sede principal da cidade de Quito. Além disso, deixou-se aberta a possibilidade de que pessoas de fora da universidade pudessem participar da enquete para que, dessa forma, fosse possível avaliar se o consumo de pessoas da comunidade universitária que receberam informações sobre o uso sustentável da água apresentava alguma diferença em relação àquele de pessoas que não as receberam. Descobriu-se que existe uma correlação significativa entre a PH e algumas variáveis socioeconômicas analisadas, como renda familiar, sexo e nível educacional. Concluiu-se também que a PH pode ser utilizada como um bom indicador agregado de consumo de água que ajude a notificar padrões insustentáveis de consumo, ainda que não seja recomendável sua utilização como instrumento para gestão e planificação hidrológica devido à concentração e à simplicidade da informação que apresenta.

Palavras-chave: Estimativa econômica, pegada hídrica individual, correlações de variáveis socioeconômicas, indicador agregado.

JEL: Q5 Economia ambiental.

\section{Introducción}

L

a presente investigación, de tipo exploratoria (permite estudiar un problema que no está claramente definido) y descriptiva (tiene como finalidad definir, clasificar, catalogar o caracterizar el objeto de estudio mediante el uso, por ejemplo, de encuestas) (Hernández-Sampieri y Mendoza 2018), tiene como objetivo principal el análisis y estimación volumétrica de la huella hídrica personal $(\mathrm{HH})$ para un estudio de caso concreto en Ecuador y determinar la existencia de correlaciones entre esta $\mathrm{HH}$ estimada con algunas variables socioeconómicas como el sexo, la edad, el ingreso familiar, el nivel de educación, el tipo y los años de la vivienda y el número de personas con las que comparten la vivienda los encuestados.

Este trabajo resulta significativo en el marco de investigación sobre la gestión y administración eficiente de recursos naturales, debido a que este tipo de estudios en los que se analizan elementos significativos para la deter- 
minación volumétrica (metros cúbicos/per cápita/año) de la HH personal, no han sido explorados anteriormente en Ecuador, y menos aún se ha investigado en el país sobre la existencia de correlaciones entre esta $\mathrm{HH}$ personal (que hace referencia al consumo directo e indirecto de agua por parte una persona) y determinadas variables socioeconómicas (investigación de tipo exploratoria).

La hipótesis de partida de esta investigación y lo que se intenta comprobar es la existencia de correlaciones significativas entre la $\mathrm{HH}$ personal y determinadas variables socioeconómicas como las antes mencionadas. Cabe resaltar que los objetivos de esta investigación se contraponen a los resultados obtenidos por otra investigación similar realizada en Yunlin (Taiwán) en la que se sostiene que la $\mathrm{HH}$ personal y los antecedentes socioeconómicos de las personas revelaban que el sexo, la ocupación, la edad, el nivel educativo y los ingresos anuales no afectan a las $\mathrm{HH}$ individuales, lo que indicaría, según este estudio, que la $\mathrm{HH}$ no estaría relacionada con el contexto socioeconómico (Lee et al. 2016). Esta contraposición de estudios que, si bien se desarrollan en contextos socioeconómicos diferentes, es una contribución al conocimiento y sobre todo abre la posibilidad de que se sigan profundizando las investigaciones en un ámbito de significativa importancia para el futuro como es la gestión sostenible de un recurso natural escaso y fundamental para la vida: el agua dulce.

La escasez de agua dulce es una realidad que afecta a todas las regiones del mundo en mayor o menor escala, y puede tener importantes consecuencias en la calidad de vida de las personas y el crecimiento económico de los países. En definitiva, la posibilidad de contar con fuentes de agua dulce seguras es esencial para la vida, pero también para el bienestar de la población de un país.

Si se tiene en cuenta que el volumen total de agua en la Tierra se estima en 1400 millones de kilómetros cúbicos $\left(\mathrm{km}^{3}\right)$, y que tan solo el 2,5\% de este volumen es agua dulce $\left(35\right.$ millones de $\left.\mathrm{km}^{3}\right)$, da una señal clara del problema ambiental. De esta cantidad total de agua dulce, el 69,7\%, es decir, 24,3 millones de $\mathrm{km}^{3}$ está en forma de hielo en los polos (Antártida y el Ártico), el $30 \%\left(10,5\right.$ millones de $\left.\mathrm{km}^{3}\right)$ se encuentra contenida en acuíferos subterráneos y tan solo alrededor del $0,3 \%\left(0,1\right.$ millones de $\left.\mathrm{km}^{3}\right)$ forma parte de lo que se denomina agua dulce superficial disponible para el uso de los huma- 
nos y demás seres vivos que forman parte del ecosistema (Boggiano 2015).

Ecuador, si bien es un país que cuenta con importantes fuentes y reservas de agua dulce, no es menos cierto que los fenómenos globales como el cambio climático obliga a tomar una mayor conciencia sobre el uso responsable de los recursos escasos, entre ellos, el agua dulce. Tomando en cuenta los resultados de la aplicación del indicador denominado Total Actual Renewable Water Resources (TARWR) (FAO 2003), se puede determinar que el Ecuador es uno de los países que cuenta con mayores reservas de agua dulce en el ámbito mundial, especialmente si se toma en cuenta su población (per cápita). Ecuador ocupa el puesto 27 de un ranking a nivel mundial con un volumen de agua total medido en $\mathrm{km}^{3}$ / año estimados para 2014 (FAO 2016).

Para llevar a cabo esta investigación e intentar demostrar la existencia de correlaciones entre la $\mathrm{HH}$ y determinadas variables socioeconómicas, se realizó una encuesta virtual a finales de 2019 a toda la comunidad universitaria (docentes, administrativos y alumnos) de la Pontificia Universidad Católica del Ecuador (PUCE) en la sede principal de la ciudad de Quito. También se dejó abierta la posibilidad de que personas externas a la comunidad universitaria pudieran llenar la encuesta y, de esta manera, evaluar si el consumo de los miembros de la comunidad universitaria, que habían recibido información sobre uso sustentable del agua, presentaba alguna diferencia con las personas que no la habían recibido. Con los resultados de esta encuesta y al amparo de la teoría relevante que sobre la $\mathrm{HH}$ se ha desarrollado en los últimos años, se espera contribuir a despejar algunas de las dudas sobre las interrelaciones entre el consumo y contaminación del agua y los factores socioeconómicos determinantes de dicha demanda.

\section{Aproximación teórica a la huella hídrica}

El estudio de la $\mathrm{HH}$ y su concepto fueron introducidos por Arjen Hoekstra en 2002 y desde entonces ha sido difundido por la organización Water Footprint Network (WFN) (Proyecto Huella de Ciudades 2014). No obstante, cabe mencionar que ya en 1993 Tony Allan había desarrollado el concepto de agua virtual como una manera de explicar cómo los países áridos alimentaban a su población. Es sobre este concepto primigenio de agua virtual que 
Hoekstra desarrolla sus estudios de la $\mathrm{HH}$ como un indicador para medir el consumo y la contaminación de agua dulce que contempla las dimensiones de la $\mathrm{HH}$ directa e indirecta. La $\mathrm{HH}$ mide el volumen de agua consumida para producir bienes y servicios a lo largo de la cadena de producción incluyendo la medida de agua virtual incorporada a la producción de los productos (WFN 2016).

La HH es un indicador multidimensional que presenta los volúmenes de agua consumida por fuente de consumo y los volúmenes de agua contaminada por tipo de contaminante (HH directa), todos los componentes (variables) de la HH pueden ser especificados geográfica y temporalmente y se podrían definir de la siguiente manera (Hoekstra et al. 2011).

- Huella hídrica azul: hace referencia al consumo de los recursos hídricos azules (agua dulce), superficial o subterránea, en toda la cadena de producción. El consumo se refiere a la utilización (uso o pérdida) de agua de las masas o cuerpos de aguas superficiales o subterráneas en el área de la cuenca. La pérdida ocurre cuando el agua se evapora, no regresa a la misma cuenca, es dispuesta al mar o se incorpora a un producto.

- Huella hídrica gris: hace referencia a la contaminación del agua y está definida como el volumen de agua dulce que se requiere para asimilar unas cargas contaminantes dadas las concentraciones naturales y estándares ambientales de calidad del agua.

- Huella hídrica verde: hace referencia al consumo de recursos de agua verde, es decir, agua de lluvia que no se convierte en escorrentía, sino que se incorpora en productos agrícolas.

Por otro lado, la $\mathrm{HH}$ indirecta engloba dentro de su evaluación a los tres tipos de huellas mencionados anteriormente, y se refiere al volumen de agua incorporada o contaminada en toda la cadena de producción de un producto. Para citar un ejemplo en el que se pueda apreciar de mejor manera la $\mathrm{HH}$ indirecta, se puede presentar la producción de cerveza, representada en la figura 1. En primer lugar, durante el crecimiento de la cebada utilizada en su producción se ha consumido y contaminado agua. En segunda instancia, se ha utilizado y contaminado agua durante el proceso de producción en sí mismo. En tercera instancia, se ha usado y consumido agua (aunque en menor medida) en el proceso de comercialización del producto terminado, hasta que finalmente el producto, en este caso la cerveza, llega al consumidor final. 
Figura 1

\section{La huella hídrica directa e indirecta}

H. H. indirecta

Sector
agropecuario:
cultivo de
cebada

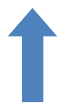

H. H. directa (verde, azul y gris)
H. H. indirecta
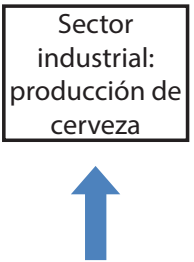

H. H. directa (azul y gris)
H. H. indirecta

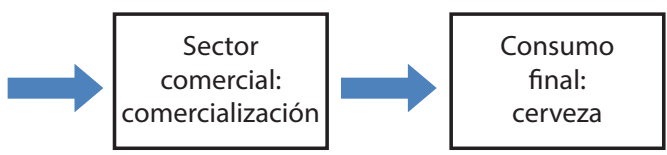

H. H. directa

(azul y gris)

Elaboración propia.

De manera esquemática, se puede representar igualmente a la $\mathrm{HH}$ de un productor o consumidor tomando en cuenta los diferentes elementos analizados anteriormente. En la figura 2, se ofrece una representación esquemática de los componentes que forman parte de la $\mathrm{HH}$, en ella se muestra que los usos no consuntivos que forman parte del agua captada y que constituyen el flujo de agua que retorna a la cuenca, no es parte de la HH. Adicionalmente, se presenta que más allá de las medidas tradicionales del uso y extracción del agua que son contempladas en las estadísticas oficiales que miden la utilización del recurso, la $\mathrm{HH}$ de un productor o un consumidor incluye $\mathrm{HH}$ verde, la $\mathrm{HH}$ gris y el uso indirecto del agua. 
Figura 2

Representación de los componentes de la huella hídrica

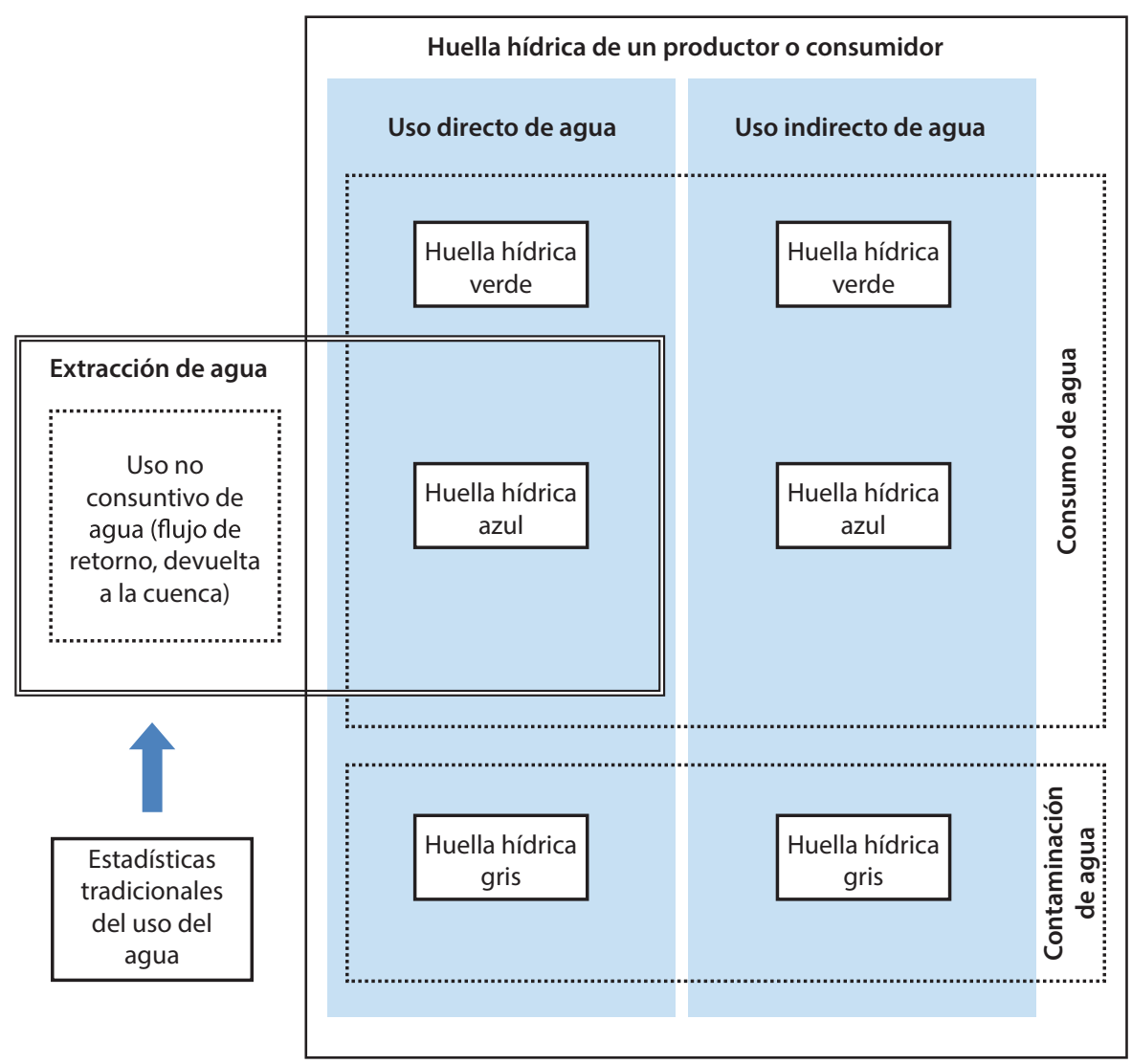

Fuente: adaptado de Hoekstra et al. (2011).

En tal sentido, se puede afirmar que la $\mathrm{HH}$ es un indicador del uso de agua que difiere de las medidas tradicionales de extracción del recurso en tres aspectos fundamentales (Hoekstra et al. 2011).

1. No incluye el agua azul que es devuelta a la cuenca.

2. No está restringida al uso de agua azul ya que incluye el agua verde y gris. 
3. No está restringida al uso directo del agua porque incluye el uso indirecto de la misma.

Es así que la $\mathrm{HH}$ ofrece una amplia perspectiva de como los consumidores y productores de bienes y servicios hacen uso de un recurso escaso como es el agua dulce, por ser una medida volumétrica del uso y la contaminación del agua. No obstante, hay que tener presente que la $\mathrm{HH}$ no es una medida de la severidad de los impactos ambientales ocasionados por el uso o la contaminación del agua en sí misma, ya que las intensidades de dichos impactos dependerán de otros factores como la cantidad estimada de los recursos, la calidad y la vulnerabilidad de los sistemas hídricos, que son propios de cada región.

Hay que tener presente que la simpleza didáctica que presenta la metodología desarrollada por la $\mathrm{HH}$ y que consiste en resumir en una sola medida volumétrica el consumo total de agua necesaria para los procesos de producción y consumo de las personas, industrias, ciudades y cuencas hidrográficas, es una idea absolutamente seductora, y que mal enfocada puede llevar a tomar decisiones equivocadas por parte de los hacedores de política pública que la quieran utilizar como medida - incluso de penalización- en el comercio internacional mediante la aplicación de medidas arancelarias a países o industrias poco sostenibles, por lo que siempre resultará necesario complementarlo con otros indicadores para que sea adecuadamente contextualizado (Chapagain y Tickner 2012).

Por lo tanto, la $\mathrm{HH}$ debe ser analizada como lo que es, un indicador agregado del consumo de agua que puede ser útil para medir la evolución de estos consumos a lo largo del tiempo y comprobar qué patrones insostenibles de uso del recurso se han modificado, tal vez mediante la mayor toma de conciencia de la población. En este sentido, la $\mathrm{HH}$ puede ser utilizada como un indicador de seguimiento que ofrezca información sobre los avances en temas de eficiencia, equidad, sustentabilidad y responsabilidad social de una población determinada (Llop et al. 2017).

También se debe tener mucho cuidado con usar la HH para realizar análisis comparativos entre determinados países o regiones, en los que nuevamente la contextualización es necesaria tener en cuenta, ya que la estimación de la $\mathrm{HH}$ de personas que viven en regiones del África subsahariana con evidentes problemas de estrés hídrico, podrían arrojar indicadores de $\mathrm{HH}$ 
altamente sostenibles por presentar volúmenes bajos de consumo per cápita, lo que sería un error.

De igual manera, al tratarse de un indicador agregado hay que tener presente que se pueden dar casos de doble contabilidad en la suma de los consumos de agua y solapamientos entre el agua verde y azul que forma parte de la HH. Es decir, la HH es un indicar sencillo que no se le puede pedir más de lo que puede explicar. Antes de continuar con el análisis de la $\mathrm{HH}$ resulta necesario explicar brevemente un concepto que estaba pendiente y es el de agua virtual.

\section{La huella hídrica y el agua virtual}

Desde la perspectiva del uso y aprovechamiento del agua en un contexto de intercambio de bienes y servicios entre diferentes regiones, el concepto de agua virtual tiene significativa importancia principalmente para las regiones que sufren mayores niveles de estrés hídrico y que tienen que obtener los productos que necesitan de otras regiones generalmente superavitarias en este recurso.

En este sentido, resulta interesante conocer la cantidad de agua contenida en los productos que exporta una determinada región o país y el agua que a su vez importa de manera virtual en los productos que llegan del exterior para cubrir las necesidades de la población. En definitiva, lo que interesa conocer es el denominado balance de agua virtual producto del comercio de productos y servicios en un área determinada (Garrido et al. 2010).

El balance de agua virtual en un área geográfica determinada en un período podría ser definido como la importación neta de agua virtual en dicho período $\left(V_{i, n e t}\right)$, el cual se podría representar como la importación bruta de agua virtual $\left(V_{i}\right)$, menos la exportación bruta de agua virtual $\left(V_{e}\right)$ (Hoekstra et al. 2011).

$$
V i, \text { net }=V i-V e \quad(\text { Volumen/tiempo })
$$


Un balance positivo de agua virtual implica una entrada de agua virtual al área de otras regiones con las que mantiene intercambios comerciales; un balance negativo significa un flujo de salida de agua virtual a otras regiones superior al agua virtual que ingresa al territorio analizado.

El comercio internacional de productos podría resultar en un sistema ahorrador de agua a nivel global si los bienes que son intensivos en el uso de agua son comercializados (exportados) desde regiones con alta productividad (eficiencia) en el uso de agua, hacia regiones con baja productividad en el uso del recurso, lo que resultaría en bienes con un bajo contenido de agua virtual; pero, solo un pequeño volumen de las transacciones de agua virtual a nivel internacional se debería a razones relacionadas con la escasez del recurso, ya que se basan (principalmente para los bienes agrícolas que son los que mayor cantidad de agua consumen) en factores tales como la disponibilidad de tierras de cultivo, trabajadores, tecnología, costos del comercio internacional, políticas y acuerdos nacionales e internacionales de comercio (Hoekstra y Chapagain 2008).

\section{La huella hídrica de un consumidor $y$ un producto}

La HH de un consumidor o HH personal puede ser definida como el volumen total de agua dulce consumida y contaminada para la producción de todos los bienes y servicios que un consumidor necesita para satisfacer sus necesidades en un período determinado. En este caso, la HH hídrica de un grupo de consumidores puede ser interpretada como la agregación de la $\mathrm{HH}$ de los consumidores individuales.

Para el caso de un consumidor, la $\mathrm{HH}\left(\mathrm{HH}_{\text {cons }}\right)$ puede ser calculada añadiendo a la $\mathrm{HH}$ directa $\left(\mathrm{HH}_{\text {cons, dir }}\right)$ del consumo del individuo, la $\mathrm{HH}$ indirecta del mismo $\left(\mathrm{HH}_{\text {cons, indir }}\right)$, como ya se había señalado anteriormente (Hoekstra et al 2011).

$$
\text { HHcons }=\text { HHcons, dir }+ \text { HHcons, indir } \quad(\text { Volumen/Tiempo })
$$


La HH directa, hace referencia al uso y la contaminación del agua por parte de un consumidor en su vida diaria, por ejemplo, en su hogar o su jardín. La HH indirecta se refiere al volumen de agua consumida y contaminada necesaria para la producción de bienes y servicios que necesita el mencionado consumidor, como, por ejemplo, comida, ropa, papel, energía, bienes industriales, entre otros. $\mathrm{La} \mathrm{HH}$ indirecta puede ser estimada multiplicando todos los productos consumidos por el individuo, por la respectiva $\mathrm{HH}$ de cada uno de dichos productos.

\section{HHcons, indir $=\Sigma p(C[p] x \operatorname{HHprod}[p]) \quad($ Volumen/Tiempo $)$}

Donde $\mathrm{C}[\mathrm{p}]$ representa el consumo de un producto $p$ medido en (unidades de producto/tiempo), y $H_{\text {prod }}[p]$ es la huella hídrica del producto $p$ medido en volumen de agua/unidad de producto. El conjunto de productos consumidos por un individuo representará el HH indirecta de un consumidor.

Considerando un proceso sencillo de producción de un producto determinado $p$, se pude formalizar que la $H H_{\text {prod }}[p]$, y como ya se había mencionado anteriormente, sería igual a la suma de las $\mathrm{HH}$ de los diferentes componentes del proceso productivo (HH directa y HH indirecta), dividida para cantidad total de la producción del bien $p$ :

$\operatorname{HHprod}[p]=(\Sigma s=1 \mathrm{HHproc}[s]) / P[p]($ Volumen/Unidades de producto $)$

En donde $H . H_{\text {. }}[s]$ s] es la huella hídrica del proceso en el apartado s, es decir, en una fase determinada del proceso productivo o cadena de producción, medido en volumen de agua/tiempo, y $P[p]$ es la cantidad de producción generada medida en unidades del producto/tiempo.

\section{Metodología}

\section{La huella hídrica personal en la PUCE}

Los esfuerzos por aproximarse al estudio de la $\mathrm{HH}$ en Ecuador han sido limitados y se han encontrado estudios a nivel de microcuenca (Lala y Fer- 
nández 2020) o nivel global del consumo de agua de una institución púbica como es la Secretaría del Agua (Guamán 2018), pero en ningún caso a nivel de la estimación de la HH de consumidores individuales.

Para una primera aproximación a la estimación de la $\mathrm{HH}$ personal en el caso ecuatoriano, se utilizó una metodología de investigación de tipo exploratoria ya que trata sobre un tema que se ha estudiado muy poco en Ecuador y de tipo descriptiva en la medida que permitirá caracterizar y obtener mayor información sobre esta temática novedosa y de significativa importancia para el país. Para este trabajo, se ha tomado como estudio de caso a la comunidad universitaria de la PUCE, sede central Quito, y se aplicó la metodología de estimación de HH desarrollada por la organización WFN (2017), pero con una serie de preguntas adaptadas a la realidad social y económica del país.

En este sentido, se utilizaron la mayoría de los algoritmos de cálculo desarrollados por WFN para la estimación de la HH personal, pero con modificaciones y preguntas adicionales que permitieron aproximarnos a una valoración más ajustada a los consumidores ecuatorianos; se eliminaron de la evaluación, por ejemplo, preguntas relacionadas al consumo de agua en piscinas en las viviendas (poco comunes en la realidad ecuatoriana) y se incluyeron otras como la cantidad de agua embotellada que se consume al día. Igualmente, y para mejor entendimiento de las preguntas, las unidades de medida para el consumo de alimentos fueron adaptadas cambiando de unidades de peso $(\mathrm{kg})$ a tazas o porciones, manteniendo, claro está, la misma equivalencia (ejemplos: 1 porción individual de pasta o arroz cocidos equivale en promedio a $215 \mathrm{~g} ; 1$ porción mediana (alrededor de $200 \mathrm{~g}$ ), equivale a dos rodajas de melón o sandía, una tajada de piña, una taza de fresas, uvas, cerezas). Cabe mencionar que se realizaron alrededor de 20 encuestas como prueba piloto con estudiantes y docentes de la universidad y se validó teóricamente la encuesta con varios docentes de la PUCE, especialistas en estadística, econometría e ingeniería sanitaria, para asegurarse de que las adaptaciones realizadas a las encuestas eran adecuadas y podían ser debidamente entendidas por los participantes. Aunque las fórmulas en sí no son complicadas, la dificultad radica muchas veces en encontrar los datos necesarios para aplicarlas (la encuesta utilizada se encuentra disponible en el siguiente enlace: https://www.puce.edu.ec/sitios/forms/encuesta/huella-hidrica/). 
Dentro de la encuesta, además de las preguntas que permitían estimar el consumo de agua personal $(\mathrm{HH})$, medido en término de metros cúbicos $\left(\mathrm{m}^{3}\right)$ año, se incluyeron una serie de variables socioeconómicas tales como: sexo, edad, ingreso familiar (percepción de ingreso medido de manera indirecta con escala subjetiva, ejemplo, usando respuesta como "Medio alto" o "Medio bajo") y de manera objetiva en otra pregunta, ejemplo, usando respuestas con rangos como "500 a 1000 dólares / mes o 1000 a 1500 dólares / mes"), nivel de educación, tipo de vivienda (casa o apartamento), años de construcción de la vivienda y número de personas con las que comparte la vivienda (número de adultos y niños).

Con esta metodología adaptada se realizó una encuesta en línea a los docentes, administrativos y alumnos de la PUCE en octubre de 2019, para lo cual se realizó, en primer lugar, una campaña interna (vía correo electrónico y redes sociales) de concientización sobre lo que significa la $\mathrm{HH}$ dentro de la universidad, campaña que se apoyó en otras iniciativas universitarias de (concientización ambiental y género) para lograr un campus más sostenible, bajo el lema de "Deja tu huella". Cabe mencionar que no se realizó, por parte de la PUCE, un análisis del ROI social (return on investment) de la campaña global de concientización para determinar su eficacia; no obstante, dicha campaña fue ampliamente difundida dentro de la PUCE llegando a la totalidad de los miembros de la comunidad universitaria por medio de sus correos electrónicos, redes sociales y afiches informativos colocados en lugares estratégicos del campus. También, a través de todos los medios digitales de la universidad y de la Facultad de Economía e Ingeniería (Facebook, Twitter y Linkedin) se realizó una campaña de difusión de la encuesta y se invitó a la comunidad universitaria y a personas externas a la PUCE (principalmente gente con interés en la información de la PUCE: exalumnos, familiares y amigos de los miembros de la comunidad universitaria) a participar de la iniciativa rellenando el cuestionario sobre su consumo de agua. Fue también importante el apoyo que se contó para esta investigación de las asociaciones de estudiantes y docentes que también invitaron a sus miembros a participar.

Tomando en cuenta que el número total de personas que forman parte de la comunidad universitaria en la sede Quito suman los 16.798 individuos repartidos entre 14.151 estudiantes, 1945 docentes y 702 administrativos, se estimó que la muestra necesaria para poder obtener resultados estadística- 
mente significativos con el $95 \%$ de confianza es de 376 encuestas repartidas entre 316 estudiantes, 43 docentes y 16 administrativos representados en la tabla 1. E1 tamaño de la muestra se estimó utilizando la siguiente fórmula:

$$
\mathrm{n}=\mathrm{N} \times \mathrm{Z}_{\mathrm{a}}^{2} \times \mathrm{p} \times \mathrm{q} / \mathrm{d}^{2}(\mathrm{~N}-1)+\mathrm{Z}_{\mathrm{a}}^{2} \times \mathrm{p} \times \mathrm{q}
$$

En donde:

$\mathrm{n}=$ tamaño de muestra que queremos calcular.

$\mathrm{N}=$ tamaño de la población.

$\mathrm{Z}=$ coeficiente de confianza para un nivel de confianza determinado.

$\mathrm{p}=$ probabilidad de éxito.

$\mathrm{q}=$ probabilidad de fracaso.

$\mathrm{d}=$ error máximo admisible.

Tabla 1

Comunidad universitaria, muestra y número de encuestas válidas

\begin{tabular}{|l|c|c|c|c|}
\hline $\begin{array}{c}\text { Comunidad universitaria } \\
\text { (sede Quito) }\end{array}$ & $\begin{array}{c}\text { Total } \\
\text { personas }\end{array}$ & $\begin{array}{c}\text { Porcentaje } \\
(\%) \\
\text { sobre el total }\end{array}$ & $\begin{array}{c}\text { Muestra 95\% } \\
\text { de confianza }\end{array}$ & $\begin{array}{c}\text { Número de } \\
\text { encuestas } \\
\text { válidas }\end{array}$ \\
\hline Estudiantes & 14.151 & 84 & 316 & 418 \\
\hline Docentes & 1945 & 12 & 43 & 86 \\
\hline Administrativos & 702 & 4 & 16 & 53 \\
\hline Total & 16.798 & 100 & 376 & 557 \\
\hline
\end{tabular}

Fuente y elaboración propia.

Todas las encuestas que entraron en la aplicación en línea, creada para el efecto, y que tuvieron como lapso de tiempo para el ingreso del 1 al 31 de octubre de 2019, fueron sistematizadas y analizadas por el equipo de investigación. Como se puede observar, el número de encuestas válidas obtenidas (557 encuestas) luego de realizar un proceso de depuración de la base de datos (se descartaron 46 encuestas que presentaban datos incongruentes y sesgados), supera a la cantidad de observaciones necesarias estimadas para obtener resultados estadísticamente significativos (376 encuestas). Se registraron 418 encuestas de estudiantes, 86 de docentes y 53 de personal ad- 
ministrativo. Cabe mencionar que se obtuvieron igualmente 272 encuestas válidas de personas que no pertenecen a la PUCE (externos) y que decidieron voluntariamente responder a la encuesta porque se enteraron de la misma a través de las redes sociales o fueron motivados a participar por miembros de la comunidad universitaria.

Si bien es cierto, esta muestra total de encuestas tanto de la comunidad universitaria y de personas externas (829 encuestas válidas en total) presenta un evidente sesgo de autoselección, no es menos cierto que para los objetivos de esta investigación, que es el análisis de la correlación del consumo personal de agua medido a través de la huella hídrica con determinadas variables socioeconómicas, resulta suficiente.

\section{Resultados y discusión}

El valor promedio estimado de la $\mathrm{HH}$ personal en el total de la muestra analizada (PUCE y externos), alcanzó los $861,5 \mathrm{~m}^{3} /$ per cápita / año, que no se aleja significativamente de valores promedio estimados en el ámbito mundial, utilizando la misma metodología desarrollada por WFN, que se referencian en cantidades de alrededor de $1240 \mathrm{~m}^{3}$ / per cápita / año (Hoekstra y Chapagain 2006). No obstante, al ser la HH personal estimada en esta investigación un $31 \%$ menor que el promedio mundial, alienta a pensar que se está teniendo un consumo relativamente responsable dentro de la comunidad universitaria, tomando en cuenta todas las limitaciones y restricciones que un análisis simplificado, como el de los promedios, puede aportar y que se realizaron algunas modificaciones en las preguntas utilizadas en la encuesta para adaptarla al contexto ecuatoriano.

Con toda esta información contenida en una base de datos, se procedió a la realización del siguiente análisis econométrico representado en la tabla 2 para determinar la relación existente entre la $\mathrm{HH}$ y las variables socioeconómicas anteriormente referenciadas. 
Tabla 2

Huella hídrica personal y variables socioeconómicas

\begin{tabular}{|c|c|c|c|c|}
\hline & (1) & $(2)$ & (3) & (4) \\
\hline & Toda la muestra & $\begin{array}{l}\text { PUCE vs. } \\
\text { externos }\end{array}$ & PUCE & Externos \\
\hline \multirow{2}{*}{$\begin{array}{c}\text { Sexo }(0=\text { masculino, } \\
1=\text { femenino })\end{array}$} & $-54,22^{* *}$ & $-54,47^{\star *}$ & $-66,39^{* *}$ & $-41,67$ \\
\hline & $(26,63)$ & $(26,66)$ & $(30,58)$ & $(51,03)$ \\
\hline \multirow{2}{*}{ Edad } & 2128 & 2175 & 0,577 & 3657 \\
\hline & $(1787)$ & $(1626)$ & $(2676)$ & $(2470)$ \\
\hline \multicolumn{5}{|c|}{ Nivel de educación ( 0 = primaria / secundaria) } \\
\hline \multirow{2}{*}{ Tercer nivel } & 35,38 & 32,49 & 17,70 & 56,10 \\
\hline & $(41,28)$ & $(40,04)$ & $(57,96)$ & $(59,22)$ \\
\hline \multirow{2}{*}{ Cuarto nivel } & 88,53 & $108,2^{\star \star}$ & $170,2^{*}$ & 11,58 \\
\hline & $(62,86)$ & $(48,78)$ & $(86,91)$ & $(76,26)$ \\
\hline \multicolumn{5}{|c|}{ Comunidad $(0=$ administrativo $)$} \\
\hline \multirow{2}{*}{ Docente } & 79,19 & & 30,77 & \\
\hline & $(71,50)$ & & $(76,50)$ & \\
\hline \multirow{2}{*}{ Estudiante } & 35,99 & & 34,38 & \\
\hline & $(69,60)$ & & $(88,80)$ & \\
\hline \multirow{2}{*}{ Externo } & 23,78 & & & \\
\hline & $(63,64)$ & & & \\
\hline \multicolumn{5}{|c|}{ Ingreso familiar ( 0 = bajo) } \\
\hline \multirow{2}{*}{ Medio bajo } & 73,54 & 76,84 & $176,4^{* * *}$ & $-61,04$ \\
\hline & $(97,13)$ & $(97,26)$ & $(61,17)$ & $(219,7)$ \\
\hline \multirow{2}{*}{ Medio alto } & $169,2^{*}$ & $173,3^{*}$ & $311,3^{* * *}$ & $-52,12$ \\
\hline & $(96,16)$ & $(96, .25)$ & $(62,38)$ & $(213,2)$ \\
\hline \multirow{2}{*}{ Alto } & $313,7^{* *}$ & $321,9^{* *}$ & $369,9^{* *}$ & 415,1 \\
\hline & $(152,4)$ & $(150,7)$ & $(147,3)$ & $(335,1)$ \\
\hline \multirow{2}{*}{ n. ${ }^{\circ}$ adultos en el hogar } & 4,847 & 4,743 & 0,706 & 22,18 \\
\hline & $(9,802)$ & $(9,815)$ & $(12,02)$ & $(17,79)$ \\
\hline \multirow{2}{*}{ n. ${ }^{0}$ niños en el hogar } & $45,19^{* \star *}$ & $44,61^{* * *}$ & $53,23^{* * *}$ & 27,26 \\
\hline & $(13,97)$ & $(13,86)$ & $(16,78)$ & $(24,44)$ \\
\hline \multirow{2}{*}{$\begin{array}{l}\text { Años de la vivienda } \\
\text { familiar }\end{array}$} & $-0,707$ & $-0,726$ & 0,106 & $-3,669^{* *}$ \\
\hline & $(1,224)$ & $(1,223)$ & $(1,442)$ & $(1,760)$ \\
\hline \multirow{2}{*}{$\begin{array}{c}\text { Tipo de vivienda }(0= \\
\text { casa, } 1=\text { apartamento) }\end{array}$} & $-26,76$ & $-27,73$ & $-27,02$ & $-6,351$ \\
\hline & $(28,63)$ & $(28,30)$ & $(33,25)$ & $(57,34)$ \\
\hline
\end{tabular}




\begin{tabular}{|c|c|c|c|c|}
\hline & $(1)$ & $(2)$ & $(3)$ & $(4)$ \\
\hline \multirow{2}{*}{ PUCE } & Toda la muestra & $\begin{array}{c}\text { PUCE vs. } \\
\text { externos }\end{array}$ & PUCE & Externos \\
\hline \multirow{2}{*}{ Constante } & & 12,64 & & \\
\cline { 2 - 5 } & $(135,9)$ & $(33,03)$ & & \\
\hline Observaciones & 845 & $648,4^{* * *}$ & $547,5^{* * *}$ & $778,7^{* * *}$ \\
\hline R-cuadrado & 0,074 & 0,073 & $(158,8)$ & $(223,6)$ \\
\hline
\end{tabular}

t de Student entre paréntesis

${ }^{* * *} p<0,01,{ }^{* *} p<0,05,{ }^{*} p<0,1$

Fuente: Base de datos, encuesta de huella hídrica.

Elaboración propia.

En la tabla 2, entre paréntesis, se muestran los valores del estadístico t de Student y los asteriscos (*) muestran la significancia estadística individual (p-valor) asociada a la estimación de cada parámetro. De igual forma, en la parte inferior se muestra la medida de bondad de ajuste expresada a través del estadístico R-cuadrado.

Para el análisis de resultados se realizaron 4 regresiones, la primera (1) con toda la muestra donde se encuentran miembros y no miembros de la PUCE, la segunda (2) observando si es que existe alguna diferencia entre miembros y no miembros de la PUCE, la tercera (3) estimando los efectos solamente para miembros de la PUCE y la cuarta (4) a través de la cual se buscó determinar los factores que más influyen en la huella hídrica producida por una persona externa a la PUCE.

De acuerdo con la primera estimación (1) los factores que más influyen (Ceteris Paribus) para la huella hídrica personal son el sexo, puesto que el sexo femenino está asociado con un consumo en promedio 54,22 $\mathrm{m}^{3}$ de agua en un año, menor frente al sexo masculino, lo que podría indicar un mayor grado de sensibilidad y concientización sobre el consumo responsable del sexo femenino frente al masculino (hipótesis que habría que verificar en posteriores estudios). Otra variable estadísticamente significativa son los ingresos familiares, puesto que aquellas personas cuyos ingresos familiares son medio-altos o altos se asocian con un mayor consumo de agua con valo- 
res de 169,2 y $313,7 \mathrm{~m}^{3}$ de agua en un año en promedio respectivamente con relación a los hogares de ingresos bajos.

El hecho de que las personas que se identifiquen con un mayor ingreso familiar promedio tengan mayores consumos promedio de agua estaría en concordancia con la interpretación microeconómica del agua como un bien normal, es decir que aumenta su consumo cuando aumenta la renta. Finalmente, la última variable con la cual se encontró correlación es el número de niños que viven en el hogar, lo cual hace que el consumo de agua se eleve en aproximadamente $45 \mathrm{~m}^{3}$ por año en promedio (Ceteris Paribus), el cual es un resultado esperado ya que la atención y cuidado de los niños demanda un mayor consumo de agua en el ámbito individual y familiar.

Al realizar el análisis de miembros de la PUCE en comparación con personas externas (2) se encontraron resultados similares (Ceteris Paribus), el hecho de ser de sexo femenino se asocia con un consumo de $54,47 \mathrm{~m}^{3} \mathrm{de}$ agua en un año, menor frente al sexo masculino. En este caso, se observa que aquellas personas con educación de cuarto nivel consumen en promedio $108,2 \mathrm{~m}^{3}$ de agua más al año respecto a aquellas personas con un nivel educativo de primaria/secundaria. De igual manera los ingresos familiares son determinantes respecto al consumo de agua, las personas de ingresos medio-altos y altos se encuentran asociadas con un consumo mayor en promedio de 173,3 y $321,9 \mathrm{~m}^{3}$ de agua al año respectivamente frente a las personas de hogares con ingresos bajos. El número de hijos también muestra una mayor incidencia en el consumo de agua con un aumento de $44,61 \mathrm{~m}^{3}$ al año por cada niño adicional manteniendo las demás variables constantes.

Al realizar el análisis de la muestra solamente de personas pertenecientes a la PUCE se encuentra que el sexo femenino se asocia con un coeficiente mayor en valor absoluto en el consumo de agua respecto a los hombres con una cantidad menor de $66,39 \mathrm{~m}^{3}$ al año. La variable educación muestra que una persona miembro de la PUCE con estudios de cuarto nivel consume 170,2 $\mathrm{m}^{3}$ más de agua al año aproximadamente que quienes tienen estudios de primaria/ secundaria (que en este caso son los estudiantes en su mayoría), posiblemente vinculado a unos mejores hábitos de limpieza y salud asociados al consumo de agua (hipótesis igualmente sujeta a verificación en futuros estudios).

En este grupo de personas (miembros de la PUCE) el nivel de ingresos es determinante para el consumo de agua; se observa que a medida que los 
ingresos familiares aumentan, también lo hace el consumo anual de agua, las personas con ingresos medio-bajos respecto a quienes tienen ingresos bajos consumen $176,4 \mathrm{~m}^{3}$ de agua más aproximadamente al año, quienes gozan de ingresos medios altos consumen $311,3 \mathrm{~m}^{3}$ al año más con respecto a las personas de ingresos bajos, finalmente un ingreso familiar alto se asocia con un consumo de agua superior en $369,9 \mathrm{~m}^{3}$ al año respecto a las personas de ingresos bajos. La variable número de niños vuelve a hacerse presente y se observa que la presencia de un niño adicional en el hogar se asocia con un aumento de 53,23 $\mathrm{m}^{3}$ adicionales de agua al año.

Para las personas externas de la PUCE (4) se observa que a pesar de que la variable sexo no es significativa, su coeficiente mantiene el signo y una magnitud no tan alejada de las que se obtuvo en las demás regresiones. La variable número de años de la vivienda familiar cobra significancia y se asocia con una reducción promedio de $3,67 \mathrm{~m}^{3}$ aproximadamente por cada año adicional de la vivienda familiar, lo que es un resultado inesperado ya que se esperaría que las viviendas más antiguas consumieran más agua, motivo por el cual esta variable debe de ser analizada en otro contexto más específico en un futuro estudio.

Otra variable interesante para el análisis, a pesar de no ser significativa, es el tipo de vivienda, la cual mantiene su signo en todas las estimaciones (se asocia con una reducción del consumo de agua si se vive en un departamento respecto a quienes viven en casa) y su magnitud en las primeras tres regresiones refleja un consumo menor de $27 \mathrm{~m}^{3}$ por año aproximadamente.

Otro dato que merece la pena resaltar es que la $\mathrm{HH}$ de los miembros de la comunidad universitaria que alcanza en promedio los $858,3 \mathrm{~m}^{3} /$ per cápita / año, no difiere significativamente de la $\mathrm{HH}$ de las personas externas a la PUCE que alcanza los $868,1 \mathrm{~m}^{3}$ / per cápita / año, lo que indica claramente la necesidad de seguir trabajando en las campañas de concientización mejorando quizás la manera y el medio para transmitir el mensaje sobre el uso responsable y sostenible del agua que es precisamente la mayor valía que tiene este indicador agregado y es el de proporcionar información sencilla para fomentar el objetivo de alcanzar patrones de consumo sostenibles. En tal sentido, resulta conveniente volver a realizar una encuesta similar en el futuro en la universidad para comprobar, como así lo permite este indicador sencillo y práctico como es el de la $\mathrm{HH}$, que la comunidad universitaria esta 
ciertamente asimilando los conceptos de sostenibilidad que desde la universidad se están trasmitiendo continuamente con las campañas de concientización sobre el uso responsable de los recursos en un campus que espera ser catalogado como sostenible en un futuro cercano.

Estos resultados permiten, asimismo, ampliar la discusión sobre la búsqueda de mejores y más eficientes indicadores e instrumentos económicos sobre los que se debería incidir para lograr una mejor gestión y administración de los recursos escasos.

Por último, es necesario tener mucho cuidado en el sentido de querer extrapolar de manera general y directa los resultados obtenidos en esta investigación a otros lugares y contextos, ya que los mismos han sido obtenidos en un segmento específico de la población con características propias como es la comunidad de una universidad privada en la ciudad de Quito, y no podrían asimilarse directamente a otros niveles, grupos o regiones del país con diferentes características socioeconómicas e incluso climáticas. Sin embargo, en términos generales los resultados de esta investigación permitirán sacar algunas conclusiones como las que se presentan a continuación.

\section{Conclusiones}

La principal conclusión de la presente investigación es que se ha logrado cumplir con su objetivo central: comprobar la hipótesis de partida al demostrar la existencia de una correlación significativa entre determinadas variables socioeconómicas y el consumo promedio de agua expresado a través del indicador de la $\mathrm{HH}$. Si bien es cierto, este estudio se concentra en los miembros de la comunidad universitaria de la PUCE en su sede central en la ciudad de Quito, y un grupo significativo de personas externas a la universidad, aunque relacionadas a la misma, los resultados alcanzados son suficientemente representativos y significativos para sustentar la validez y pertinencia de esta investigación académica.

Partiendo de la estimación de la HH personal adaptada a las condiciones propias de la cultura y las condiciones socioeconómicas de los miembros de la comunidad universitaria de la PUCE y de las personas externas a la universidad, se ha logrado demostrar la existencia de una correlación entre el 
consumo de agua $(\mathrm{HH})$ y variables socioeconómicas claves como el sexo, el nivel de ingreso familiar, el nivel de educación y el número de personas con las que se comparte la vivienda (especialmente niños).

La existencia de dicha correlación se contrapone con los resultados de otro estudio de similares características realizado en 2016 en China y que sostenía la no existencia de correlaciones significativas entre las mismas variables socioeconómicas y la $\mathrm{HH}$ personal. En ese sentido, el estudio aquí presentado aporta a la discusión sobre las interrelaciones existentes entre el consumo directo e indirecto de agua por parte de las personas y determinadas características socioeconómicas de estos consumidores.

La utilidad práctica de este estudio, más allá de intentar aportar nuevos elementos de discusión para la gestión sostenible de un recurso escaso, es el intentar ampliar el espectro de análisis de los elementos determinantes del consumo de agua de las personas, pero, avanzando un paso más, el análisis que se ha concentrado en la actualidad, como así lo atestigua la teoría presentada en este trabajo, únicamente en la estimación volumétrica de la $\mathrm{HH}$ para personas de diferentes países, ciudades, sectores productivos y cuencas hidrográficas, pero sin una aproximación específica a las variables socioeconómicas relacionadas con estos consumos, como sí se realiza en el presente estudio. Son pocos los esfuerzos académicos realizados para intentar integrar el análisis económico de los posibles determinantes socioeconómicos del consumo y contaminación de agua dulce como parte importante de la gestión eficiente del recurso.

Se ha demostrado también, en el estudio, que las personas de sexo femenino tienen patrones de consumo más sostenibles que las personas de sexo masculino (posiblemente asociado a una mayor sensibilidad por los temas ambientales y de consumo responsable), y que de igual manera las personas con un mayor nivel de educación consumen más agua (posiblemente vinculado a unos mejores hábitos de limpieza y salud asociados al consumo de agua). El ingreso familiar igualmente ha resultado ser una variable significativa a la hora de presentar mayores consumos, lo que igualmente era esperado desde el punto de vista del análisis económico del consumidor considerando al agua como un bien normal. De igual manera, y según lo esperado, el hecho de tener niños en casa ha resultado ser una variable significativa a la hora de presentar mayores consumos de agua en términos promedio. 
Por otro lado, la investigación ha reflejado que los niveles de consumo y contaminación de agua de los miembros de la comunidad universitaria que recibieron información sobre la importancia del consumo sostenible de agua y los miembros externos de la universidad que no recibieron esa información, no difiere significativamente, lo que pone de manifiesto la necesidad de continuar impulsando las campañas de concientización y mejorando la calidad y los medios a través de los cuales se está llevando los mensajes a la comunidad universitaria. Las lecciones aprendidas en este caso podrían ser interesantes para la aplicación de mejores campañas de concientización, por ejemplo, en otras sedes de la universidad situados en provincia o extrapolados a otros centros de educación superior del país.

Finalmente, la presente investigación a la luz de las reflexiones que se desprenden de las aportaciones teóricas presentadas por varios autores, refleja la validez del indicador de huella hídrica en su real utilidad que es el presentar de una manera sencilla patrones de consumo insostenibles y la evidente necesidad de seguir profundizando en este indicador agregado como herramienta de concientización y uso responsable de un recurso natural clave para el futuro, como es el agua.

\section{Referencias}

Boggiano, Miguel. 2015. "Los 10 países con más reservas de agua en el mundo". Revista Diplomacia 129: 16-19.

Chapagain, Ashok, y David Tickner. 2012. "Water Footprint: Help or Hindrance?". Water Alternatives 5 (3): 563-581. https://bit.ly/2ZL1sMo.

Food and Agriculture Organization of the United Nations (FAO). 2003. Water Reports 23: Review of World Water Resources by Country. Accedido abril de 2020. https://bit.ly/2U6r$1 \mathrm{Vg}$.

---. 2016. Aquastat. https://bit.ly/2Mpmxow.

Garrido, Alberto M., Ramón Llamas, Consuelo Varela-Ortega, Paola Novo, Roberto Rodríguez-Casado y Maite Aldaya. 2010. Water Footprint and Virtual water Trade in Spain: Policy Implications. Nueva York: Springer.

Guamán, Juan. 2018. "Estimación de la huella hídrica para la Secretaría del Agua planta central Quito". Tesis de grado, Universidad Politécnica Salesiana, Quito. https://bit.ly/2XYyO93. 
Hernández-Sampieri, Roberto, y Christian Mendoza. 2018. Metodología de la investigación. Las rutas cuantitativa, cualitativa y mixta. Ciudad de México: McGraw-Hill.

Hoekstra, Arjen, Ashok Chapagain, Maite Aldaya y Mesfin Mekonnen. 2011. The Water Foodprint Assessment Manual. Londres: Earthscan.

Hoekstra, Arjen, y Ashok Chapagain. 2006. "Water Footprints of Nations: Water Use by People as a Function of Their Consumption Pattern". Water Resour Manage 21 (1): 35-48.

---. 2008. Globalization of Water: Sharing the Planet's Freshwater Resources. Oxford: Blackwell Publishing.

Lala, Henry, y Mirian Fernández. 2020. "Análisis de la sostenibilidad mediante huella hídrica de la microcuenca del río Pita, Ecuador”. Tecnología y ciencias del agua 11 (1): 169-234. https://doi.org/10.24850/j-tyca-2020-01-05.

Lee, Yung-Jaan, Chuan-Ming Tung, Piin-Rong Lee y Shih-Chien Lin. 2016. "Personal Water Footprint in Taiwan: A Case Study of Yunlin County". Sustainability 8 (11): 1-12; https:// doi.org/10.3390/su8111112.

Llop, Armando, Eduardo Comellas, Mauricio Buccheri, Valeria Mendoza, Patricia Puebla, Alicia Duek, Graciela Fasciolo y Armando Bertranou. 2017. "Sobre el alcance y aplicabilidad de la huella hídrica". Asociación Argentina de Economía Agraria. Accedido febrero de 2020. https://bit.ly/2U58tVx.

Proyecto Huella de Ciudades. 2014. "Manual para la evaluación de la huella hídrica”. Accedido 4 de febrero. https://bit.ly/2XprO5W.

Water Foodprint Network (WFN). 2016. Accedido enero 2020. http://waterfootprint.org/en/.

WF Calculator. 2017. Metodología de la calculadora de huella hídrica. Accedido enero de 2020. watercalculator.org: https://bit.ly/3ds0Wrn. 